\title{
LIDERANDO EQUIPES À DISTÂNCIA: UMA CONTEXTUALIZAÇÃO NECESSÁRIA SOBRE LIDERANÇA REMOTA E EQUIPES VIRTUAIS
}

\author{
Ricardo Pereira ${ }^{\mathbf{1}}$ \\ Cristiano José Castro de Almeida Cunha²;
}

\begin{abstract}
The coronavirus crisis accentuated a trend that had been occurring for some years: the adoption of remote work. In recent months, many companies have adhered to this new model that uses information and communication technologies as a means to connect leaders and followers in virtual teams. The purpose of this article is to identify the challenges of leaders and the most appropriate leadership styles to maintain the good performance of a virtual team. The research was carried out using the method of integrative literature review and articles collected in the Scopus and Web of Science databases were analyzed. The study revealed that the main challenges facing virtual team leaders are related to: communication, cooperation, establishing work rules and monitoring team work and that the literature does not indicate an ideal style of leadership in this context.
\end{abstract}

Keywords: remote leadership; virtual teams; virtual leadership; e-leadership

Resumo: A crise causada pelo coronavirus acentuou uma tendência que já vinha ocorrendo há alguns anos: a adoção do trabalho remoto. Nos últimos meses, muitas empresas aderiram a este novo modelo que usa as tecnologias da informação e comunicação como meio para conectar líderes e liderados nas equipes virtuais. $O$ objetivo deste artigo é identificar os desafios dos líderes e os estilos de liderança mais adequados para manter o bom desempenho de uma equipe virtual. A pesquisa foi realizada utilizando-se o método da revisão integrativa da literatura e foram analisados artigos coletados nas bases de dados Scopus e Web of Science. O estudo revelou que os principais desafios dos líderes de equipes virtuais estão relacionados com: comunicação, cooperação, estabelecimento de normas de trabalho e acompanhamento do trabalho da equipe e que a literatura não aponta um estilo ideal de liderança nesse contexto.

Palavras-chave: Liderança remota; equipes virtuais; liderança virtual; e-liderança

\footnotetext{
${ }^{1}$ Doutorando em Engenharia e Gestão do Conhecimento - Universidade Federal de Santa Catarina (UFSC), Florianópolis - Brasil. E-mail: rikardop@gmail.com

${ }^{2}$ Docente no programa de pós-graduação em Engenharia e Gestão do Conhecimento - Universidade Federal de Santa Catarina (UFSC), Florianópolis - Brasil. E-mail: 01cunha@ gmail.com
} 


\section{INTRODUÇÃO}

Nas últimas três décadas, o trabalho remoto tem se tornado cada vez mais popular e mudado a maneira como as organizações e as equipes operam. Esta é uma tendência que não apresenta indícios de abrandamento. Pelo contrário, com a crise causada pelo Covid-19, nos últimos meses, muitas empresas (grandes e pequenas) foram obrigadas a aderir e se adaptar ao trabalho remoto. De acordo com dados do Gallup Panel (Hickman \& Saad, 2020), nos EUA a porcentagem de adultos que trabalham em casa aumentou de $31 \%$ em meados de março para $62 \%$ em meados de abril de 2020 devido à preocupação com a Covid-19.

Várias grandes empresas como Ford, Facebook, Google e Alibaba adotaram o trabalho remoto como modelo. Devido às incertezas provocadas pelo coronavírus e algumas vantagens deste modelo, empresas, como o Twitter, já declararam que os seus colaboradores podem continuar trabalhando remotamente por tempo indeterminado (O’Mara, 2020).

Um elemento central do trabalho remoto é a equipe virtual - uma equipe dispersa geograficamente, constituída por pessoas que interagem, normalmente, por meios de comunicação eletrônica e que, portanto, têm pouca (ou nenhuma) interação face a face (Malhotra, Majchrzak, \& Rosen, 2007). São pessoas que trabalham juntas, mesmo estando geograficamente separadas (Maduka, Edwards, Greenwood, Osborne, \& Babatunde, 2018).

O modelo das equipes virtuais traz vantagens como redução de custos, aumento da produtividade, menos absenteísmo, menos poluição e menos acidentes (Picu \& Dinu, 2016; Cascio, 2000; Baruch, 2000). Por outro lado, trazem, também, alguns desafios como dificuldade de manter o comprometimento dos colaboradores, redução do contato social que pode levar a uma sensação de isolamento, e a necessidade de uma nova dinâmica de liderança (Picu \& Dinu, 2016; Cascio, 2000; Baruch, 2000). Dentre estes desafios, um dos mais importantes é como se adaptar a uma nova dinâmica de liderança. Quando adequada, a liderança de uma equipe virtual pode aumentar a produtividade e o moral; mas, se o estilo de liderança não for compatível com as novas estruturas, pode gerar conflito, desmotivar os membros da equipe e reduzir a produtividade. 
O objetivo deste artigo é identificar os desafios dos líderes e os estilos de liderança mais adequados para manter o bom desempenho de uma equipe virtual.

Além da introdução, o artigo apresenta cinco seções. As seções 2 e 3 abordam os referenciais teóricos sobre equipes virtuais e liderança à distância. A quarta seção descreve os aspectos metodológicos da pesquisa. A quinta seção traz a análise e discussão dos resultados. Por fim, a última seção apresenta as considerações finais, limitações e recomendações para trabalhos futuros.

\section{EQUIPES VIRTUAIS}

Uma das características do ser humano é buscar a socialização - estar reunido a outras pessoas. A interação contribui para a constituição de grupos de pessoas, que se agregam por interesses ou atributos comuns. Os indivíduos de um grupo se vêm e são vistos pelos outros como uma entidade social. São indivíduos interdependentes por causa das tarefas que desempenham como membros de um grupo e, enquanto grupo, são incorporados em um ou mais sistemas sociais maiores, por exemplo, uma organização (Mcgrath \&Argote, 2001)

Para se qualificarem como equipes, os membros devem interagir; devem trabalhar em direção a objetivos comuns; e devem adaptar-se às circunstâncias de modo a alcançar esses objetivos (Forsyth, 2010)

Um tipo de equipe cada vez mais importante para as organizações é a equipe virtual. Embora a definição de virtualidade seja multidimensional, as duas dimensões mais consistentemente usadas para caracterizá-la são: dispersão geográfica e a comunicação intermediada pelas tecnologias (Cascio \& Shuryaglio, 2003).

Nesse sentido, as equipes virtuais são definidas como dois ou mais indivíduos que usam uma mistura de tecnologias de comunicação e colaboração; geograficamente dispersos; têm contato pessoal limitado e trabalham de forma interdependente para alcançar objetivos comuns (Davis \& Bryant, 2003).

As equipes virtuais são contemporâneas da internet e sua exploração comercial. No final da década de noventa do século passado, já se estudava as equipes virtuais. Um dos primeiros trabalhos a tratar o tema foi o de Towsend e colegas (1998), que chamaram a atenção para as

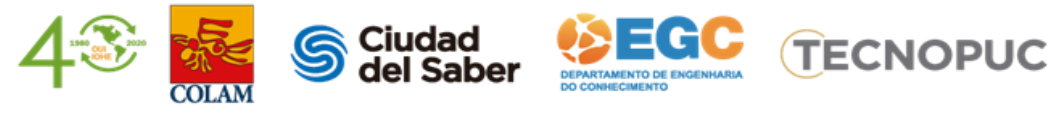


qualidades desses arranjos organizacionais. Para os autores, a popularidade das equipes virtuais nas organizações modernas é atribuída aos sucessos da estruturação do trabalho em equipe, aos avanços da tecnologia da informação e das telecomunicações, à globalização e às crescentes pressões para competir por talentos em todo o mundo, proporcionando flexibilidade e agilidade, reduzindo os custos operacionais e os custos operacionais (Townsend, DeMarie e Hendrickson, 1998).

O uso de equipes virtuais é vantajoso, pois elas podem obter resultados que as equipes presenciais não alcançariam. Alguns benefícios das equipes virtuais incluem: a possibilidade de montar equipes que maximizam o conhecimento funcional incluindo profissionais dispersos geograficamente; permitir produtividade contínua 24 horas por dia, 7 dias por semana, usando diferentes fusos horários a seu favor; reduzir custos de viagens, realocação e despesas gerais; e compartilhar conhecimento entre fronteiras geográficas e unidades organizacionais (Dulebohn \& Hoch, 2017).

\section{LIDERANDO À DISTÂNCIA}

Ao longo dos anos, a liderança foi definida e conceituada de várias maneiras. O componente comum a quase todas as definições é que a liderança é um processo de influência que auxilia grupos de indivíduos na consecução de objetivos (Northouse, 2004). É o processo de influenciar outras pessoas a entender e concordar com o que precisa ser feito e como fazê-lo (Yukl, 2010).

Liderança envolve influenciar um grupo de indivíduos que têm um objetivo comum. Pode ser um pequeno grupo de tarefas, um grupo comunitário ou um grande grupo que abrange uma organização inteira (Northouse, 2004).

Se o processo de liderança acontece no contexto de grupos e consiste em um processo de influenciar os indivíduos a atingir determinados objetivos, quando esses indivíduos formam equipes virtuais, o processo de liderança se torna mais complexo.

Em equipes virtuais, os processos de liderança são mediados por tecnologias da informação e comunicação (TICs) que funcionam como facilitadoras da comunicação, interação, e desenvolvimento e compartilhamento de tarefas (Machado \& Brandão, 2019). 
Os ambientes virtuais proporcionaram o surgimento de uma nova dinâmica de trabalho em equipe e de liderança (Avolio, Kahai, Dumdum \& Sivasubramaniam, 2001).

Equipes virtuais, em contraste com as equipes tradicionais, trabalham em locais diferentes, as tarefas são mais estruturadas e a comunicação é, em grande parte, eletrônica e assíncrona. (Kratzer, Leenders \& van Engelen , 2005). Neste novo contexto, emerge o conceito de "liderança virtual" ou "e-leadership" definida como: "o processo de influência social mediado por tecnologias avançadas da informação [Advanced Information Technology] para produzir mudanças de atitudes, sentimentos, pensamentos, comportamentos e/ou desempenho de indivíduos, grupos e/ou organizações " (Avolio, Kahai, \& Dodge, 2001b, p. 617). Os líderes de equipes virtuais devem: ser orientados para a comunicação; promover o fortalecimento da confiança; e ser capazes de criar um ambiente estável.

Apesar das pesquisas nesta área terem avançado nos últimos anos, há muito mais a ser estudado sobretudo, no que se refere a como os líderes devem atuar para manter as equipes virtuais produtivas (Garcia, 2020; Kylefalk \& Hallberg, 2020). É com este estímulo em mente que se realizou o presente estudo.

\section{PROCEDIMENTOS METODOLÓGICOS}

A metodologia utilizada foi a revisão integrativa da literatura (Botelho, Cunha, \& Macedo, 2011; Whittemore \& Knafl, 2005; Tranfield, Denyer \& Smart, 2003). Este tipo de revisão difere da revisão narrativa por adotar um processo replicável, científico e transparente de pesquisa. A revisão integrativa é uma técnica que visa minimizar o viés através de pesquisas bibliográficas exaustivas de estudos publicados, explicitando as decisões, procedimentos e conclusões dos revisores (Tranfield, Denyer \& Smart, 2003).

Tranfield, et. al. (2003) propõem três etapas para a revisão integrativa: planejamento da revisão; condução da revisão; e relatório e disseminação dos resultados da revisão.

ETAPA 1- PLANEJAMENTO DA REVISÃO: A pesquisa iniciou com a identificação da necessidade da revisão e contextualização do tema. A definição e contextualização do tema delimita e particulariza seu conteúdo evitando ambiguidade que poderia gerar desvio do foco da pesquisa (Botelho, Cunha, \& Macedo, 2011). 
Na última fase da etapa 1, foi construído o protocolo da revisão. O protocolo é um plano que contibui para a objetividade da pesquisa, através de uma descrição explícita dos passos que foram seguidos.

ETAPA 2: CONDUÇÃO DA REVISÃO: Nesta etapa, foram definidos: estratégia de busca; bases de dados; período de tempo; idiomas dos artigos; termos a serem pesquisados; ferramentas para coleta e organização das informações; e os critérios de inclusão e exclusão.

O string de busca ("remote leadership" OR "distance leadership" OR "virtual leadership" OR "e-leadership" AND "virtual tea*”) fundamentou as pesquisas nas bases de dados Scopus e Web of Science, por título, resumo e palavras-chave. Foram pesquisados artigos e revisões, sem delimitação temporal o que gerou um conjunto de publicações apresentado no quadro 1.

$\mathrm{Na}$ fase seguinte (seleção de dados), realizou-se a leitura dos resumos, palavras-chave e títulos das publicações, organizou-se os estudos relacionados e selecionou-se os estudos que compuseram o portfólio. Essa etapa foi concluída categorizando-se os estudos selecionados.

Tabela 1. Artigos selecionados por Base de Dados

\begin{tabular}{c|c}
\hline Base de dados & Número de artigos \\
\hline Scopus & 20 \\
\hline Web of Science & $(+) 22$ \\
\hline Duplicados & $(-) 13$ \\
\hline Rejeitados & $(-) 5$ \\
\hline Total de artigos selecionados & $\mathbf{2} \mathbf{2 4}$ \\
\hline
\end{tabular}

Fonte: Autores da Pesquisa (2020)

Os procedimentos de seleção dos artigos foi realizado utilizando-se a ferramenta Start (Fabbri, S. et al, 2016). Este sistema classificou os artigos de acordo com sua relevância, atribuindo scores de acordo com três critérios (palavras-chave no título, palavras-chave no resumo ou palavras-chave nas palavras-chave), além disso, os artigos foram ordenados por prioridade de leitura (very high, high, low ou very-low).

ETAPA 3: RELATÓRIOS E DISSEMINAÇÃO: O relatório foi elaborado a partir da análise e discussão dos resultados como apresentados a seguir. 


\section{ANÁLISE E DISCUSSÃO DOS RESULTADOS}

Esta seção apresenta os resultados obtidos a partir da análise e síntese dos 24 artigos relacionados no quadro 2 . O objetivo da síntese foi identificar temas que pudessem responder à questão quais os desafios dos líderes e os estilos de liderança mais adequados para manter o bom desempenho de uma equipe virtual. A síntese de dados foi operacionalizada com o auxílio da análise temática proposta por Braun e Clarke (2016). A análise temática possibilitou que os temas fossem identificados e codificados durante todo o processo de leitura e análise dos artigos.

Quadro 1. Artigos selecionados para análise e discussão

\begin{tabular}{|c|c|c|c|}
\hline Id & Ano & Autor & Título \\
\hline 01 & 2014 & Fan; Chan; Wang; Chen & $\begin{array}{l}\text { E-leadership effectiveness in virtual teams: motivation language } \\
\text { perspective }\end{array}$ \\
\hline 02 & 2003 & Davis; Bryant, & Influence at a distance: leadership in global virtual teams \\
\hline 03 & 2018 & $\begin{array}{l}\text { Maduka; Edwards; Greenwood; } \\
\text { Osborne; Babatunde }\end{array}$ & $\begin{array}{l}\text { Analysis of competencies for effective virtual team leadership in } \\
\text { building successful organisations }\end{array}$ \\
\hline 04 & 2009 & Purvanovaa; Bono & $\begin{array}{l}\text { Transformational leadership in context: Face-to-face and virtual } \\
\text { teams. }\end{array}$ \\
\hline 05 & 2003 & Cascio; Shurygailo & E-leadership and virtual teams \\
\hline 06 & 2007 & Hambley; O’Neill; Kline; & $\begin{array}{l}\text { Virtual team leadership: The effects of leadership style and } \\
\text { communication medium on team interaction styles and outcomes }\end{array}$ \\
\hline 07 & 2008 & Wakefield; Leidner; Garrison & $\begin{array}{l}\text { A Model of Conflict, Leadership, and Performance in Virtual } \\
\text { Teams }\end{array}$ \\
\hline 08 & 2014 & Avolio;Sosik;Kahai;Baker & $\begin{array}{l}\text { E-leadership: Re-examining transformations in leadership source } \\
\text { and transmission }\end{array}$ \\
\hline 09 & 2009 & Lee & E-ethical leadership for virtual project teams \\
\hline 10 & 2009 & Sarker; Sarker; Schneider & $\begin{array}{l}\text { Seeing Remote Team Members as Leaders: A Study of US- } \\
\text { Scandinavian Teams }\end{array}$ \\
\hline 11 & 2017 & Liao & Leadership in virtual teams: A multilevel perspective. \\
\hline 12 & 2013 & Korzynski & $\begin{array}{l}\text { Online social networks and leadership Implications of a new } \\
\text { online working environment for leadership }\end{array}$ \\
\hline 13 & 2014 & Ziek; Smulowitz & $\begin{array}{l}\text { The impact of emergent virtual leadership competencies on team } \\
\text { effectiveness }\end{array}$ \\
\hline
\end{tabular}




\begin{tabular}{|l|l|l|l|}
\hline 14 & 2015 & De Paoli; Ropo & $\begin{array}{l}\text { Open plan offices - the response to leadership challenges of } \\
\text { virtual project work? }\end{array}$ \\
\hline 15 & 2015 & Fernandez; Jawadi & Virtual R\&D Project Teams: From E-Leadership To Performance \\
\hline 16 & 2018 & Anoye; Kouamé & $\begin{array}{l}\text { Leadership Challenges In Virtual Team Environment } \\
\text { Paradoxical Virtual Leadership: Reconsidering Virtuality } \\
\text { Through a Paradox Lens }\end{array}$ \\
\hline 18 & 2012 & Murvanovaa; Kenda & Interventions for effectively leading in a virtual setting \\
\hline 20 & 2015 & Leduc; Guilbert; Vallery & $\begin{array}{l}\text { Virtual teams in organizations } \\
\text { actions }\end{array}$ \\
\hline 21 & 2010 & Neufeld; Wan; Fang & $\begin{array}{l}\text { Remote Leadership, Communication Effectiveness and Leader } \\
\text { Performance }\end{array}$ \\
\hline 22 & 2008 & Zimmermann; Wit; Gill & $\begin{array}{l}\text { The Relative Importance of Leadership Behaviours in Virtual and } \\
\text { Face-to-Face Communication Settings }\end{array}$ \\
\hline 23 & 2005 & Boje, D; Rhodes & $\begin{array}{l}\text { The Virtual Leader Construct: The Mass Mediatization and } \\
\text { Simulation of Transformational }\end{array}$ \\
\hline
\end{tabular}

Fonte: Os autores (2020)

A partir da análise dos artigos foram identificados os quatro temas apresentados a seguir: aspectos teóricos da liderança virtual; desafios dos líderes virtuais; estilos de liderança; e liderança e desempenho das equipes virtuais.

\subsection{LIDERANÇA VIRTUAL: UM NOVO CONSTRUTO}

No final dos anos 90, com a rápida disseminação das tecnologias da informação e comunicação (TIC's) - Internet, e-mail, videoconferência, equipes virtuais e sistemas de groupware (GDSS) -, vários autores começaram a pesquisar sobre como essas tecnologias transformariam a organização do trabalho e suas implicações nos processos de liderança (Avolio et. al, 2014).

O primeiro trabalho teórico que cunhou o termo liderança eletrônica (e-leadership) foi realizado por Avolio, Kahai, \& Dodge (2001). Os autores empregaram uma teoria de estruturação adaptativa para explicar como as tecnologias de comunicação podem interagir com 
os líderes de equipes e seus membros para produzir novas estruturas e culturas (Purvanovaa \& BONO, 2009).

Em sua revisão sobre liderança eletrônica, Avolio, Kahai \& Dodge (2001, p. 617), reforçam que: "escolhemos o termo liderança eletrônica para incorporar o novo contexto emergente para examinar a liderança" (Avolio et. al, 2014, p. 106). Os autores enfatizam a tecnologia como uma mediadora dos processos de influência social que normalmente estão associados à liderança e consideram que as "estruturas organizacionais, incluindo liderança, podem ser transformados como resultado de interações com a Tecnologia da Informação Avançada" (Avolio et. al, 2014, p. 106). Além disso, eles sugerem que "os líderes precisarão desempenhar um papel mais proativo na criação de estruturas sociais que promovam a implementação das tecnologias de informação computacionais" (Avolio et. al, 2014, p. 106).

O ambiente virtual e suas várias tecnologias de comunicação criaram um novo contexto para a liderança e o trabalho em equipe (Avolio, Kahai, Dumdum \& Sivasubramaniam, 2001). Para Avolio et. al (2014), tem-se, então, um objeto de pesquisa (liderança) no qual o contexto não apenas importa, mas é parte integrante do construto a ser estudado.

Como já foi visto na seção 2, este novo constructo, denominado "liderança eletrônica" ou "liderança virtual" (ou remota), é definido como "um processo de influência social mediado por tecnologias de informações avançadas para produzir mudanças de atitudes, sentimentos, pensamentos, comportamentos e/ou desempenho nos indivíduos, grupos e/ou organizações" (Avolio, Kahai, \& Dodge, 2001, p. 617).

\subsection{O LIIDER NA CONDUÇÃO DE EQUIPES VIRTUAIS: PRINCIPAIS DESAFIOS}

As equipes virtuais, cujos membros são geograficamente dispersos e multifuncionais, mas trabalham em tarefas interdependentes, apresentam desafios importantes do ponto de vista da liderança (Malhotra, et. al, 2007).

Devido à dispersão dos membros da equipe virtual, há uma tendência a experimentar maiores e mais diversificados conflitos em comparação com as equipes presenciais (Wakefield, , Leidner, \& Garrison, 2008). Por isso, os líderes precisam criar uma cultura de parceria e usar a persuasão para liderar com êxito. É necessário, também, desenvolver uma plataforma de 
confiança, harmonia e cooperação, que contribua para a melhoria do desempenho da equipe. Alguns elementos necessários para a criação da plataforma são: comunicação abundante, interação pessoal, respeito, honestidade, consenso, reconhecimento de interesse mútuo e coordenação comportamental (Fernandez \& Jawadi, 2015).

Outro importante desafio do líder de equipes virtuais é superar a dificuldade de coordenação das atividades da equipe. Para enfrentar essa dificuldade, Cascio \& Shuryaglio (2003) sugerem a adoção de um conjunto de ações: 1) promover estreita cooperação entre membros da equipe; 2) incentivar e reconhecer líderes emergentes; 3) estabelecer normas e procedimentos no início da formação e desenvolvimento da equipe; e 4) estabelecer limites adequados entre casa e trabalho.

Malhotra, et. al. (2007) estendem essas sugestões e propõem um conjunto de ações para que as equipe virtuais sejam bem-sucedidas: 1) estabelecer e manter a confiança através do uso da tecnologia de comunicação; 2) garantir que a diversidade seja entendida e apreciada; 3) gerenciar o ciclo de vida profissional (reuniões); 4) monitorar o progresso da equipe usando a tecnologia; 5) aumentar a visibilidade dos membros virtuais dentro da equipe e na organização; e 6) permitir que membros individuais da equipe virtual se beneficiem da equipe.

Cabe ao líder da equipe virtual fornecer orientação aos membros, usando uma abordagem compreensiva e empática. Quando se tratar de tarefas técnicas, as instruções devem ser mais específicas e os feedbacks mais constantes. No caso de trabalho criativo, cabe ao líder dar incentivos positivos e desafiar os membros da equipe como forma de estimular a criatividade. É importante que as regras de operação da equipe sejam definidas com antecedência, para facilitar a recepção das orientações do líder por parte dos liderados (Fan, Chan, Wang, \& Chen, 2014).

Nas equipes virtuais, funciona melhor o modelo de controle baseado em objetivos e metas e não por horas. Neste modelo, é importante que o líder seja competente na delegação de tarefas e na comunicação clara de expectativas. Cabe, também, a ele encorajar os liderados a controlar seus próprios tempos (Cascio \& Shuryaglio, 2003)

O estudo de Maduka et. al. (2018) reforça estudos anteriores e identifica algumas competências necessárias para o líder de equipes virtuais: feedback constante; habilidade para construir confiança; comunicação efetiva; e direções claras (dentre outras). O mesmo estudo 
propõe que ao selecionar os líderes de equipes virtuais se dê preferência àqueles com estilo mais próximo da liderança transformacional. Esse estilo, em princípio, é mais adequado para alcançar o sucesso da equipe virtual.

As competências e práticas descritas neta seção, podem ser usadas como elementos orientadores para o design de programas de desenvolvimento de líderes de equipes virtuais.

\subsection{ESTILOS DE LIDERANÇA NO CONTEXTO DE EQUIPES VIRTUAIS}

Davis \& Bryant (2003) acreditam que as teorias existentes sobre a liderança, com algumas modificações, podem ser adequadas para as equipes virtuais. Entretanto, os artigos analisados não identificaram um estilo de liderança que possa ser considerado o melhor ou o mais adequado para uma equipe virtual. Compete, portanto, a cada líder analisar as características da equipe e adaptar seu estilo de liderança de forma a atender às demandas contingenciais (Cascio \& Shuryaglio, 2003).

Nas equipes virtuais, os estilos e funções de liderança precisam se adaptar e integrar a virtualidade para serem eficazes e garantir o sucesso e o bom desempenho. A adaptação dos líderes envolve aprender a lidar com questões desafiadoras geradas por processos de comunicação não presenciais, diversidade de membros, problemas técnicos e, em alguns casos,

pressão de tempo. É importante levar esses fatores em consideração ao formular estratégias para desenvolver as competências do líder e reforçar o relacionamento entre os membros da equipe (Cascio \& Shuryaglio, 2003) .

Finalmente, um estudo realizado com 412 empregados de uma empresa multinacional, localizados em diferentes países, mostrou que os líderes de equipes virtuais devem apresentar tanto comportamentos orientados a tarefas quanto orientados a relacionamentos com maior intensidade do que em configurações face a face (Zimmermann, Wit, \& Gill, 2008).

\subsection{LIDERANÇA E O DESEMPENHO DAS EQUIPES VIRTUAIS}

Embora as definições iniciais de equipe virtual tenham enfatizado sua natureza temporária, pesquisas recentes mostram que equipes virtuais permanentes são cada vez mais

\section{TECNOPUC}


utilizadas para realizar projetos de longo prazo ou de renovação organizacional (Fernadez \& Jawadi, 2015).

Estudo realizado por Hambley, O’neill, \& Kline (2007) forneceu evidências de que os meios de comunicação têm efeitos importantes nos estilos de interação e coesão da equipe. Ao trabalhar virtualmente, a distância amplifica as disfunções. Devido às dispersões físicas, culturais e temporais entre os membros de equipes virtuais, a liderança passa a ter papel vital na eficácia da equipe (Malhotra et al., 2007; Fan, Chan, Wang, \& Chen, 2014). Nas equipes presenciais os membros podem reconhecer e solucionar problemas com mais rapidez e facilidade, especialmente problemas interpessoais. Já nas equipes virtuais, porque quase toda a comunicação é realizada à distância, e não pessoalmente, há menos oportunidades de reconhecer problemas e mais dificuldade em resolvê-los (Davis \& Bryant, 2003).

Nas equipes virtuais, os líderes exercem influência e acompanham as atividades dos liderados mediados pelas tecnologias da comunicação e colaboração (e-mail, groupware). As variáveis mediadoras influenciam os comportamentos dos líderes e liderados, em particular no que se refere ao uso adequado das TICs e às contribuições para o desempenho da equipe (Davis \& Bryant, 2003)

Em um nível mais geral, as empresas que usam equipes virtuais melhoram o desempenho, empregando indivíduos qualificados ao redor do mundo e criando times que seriam inviáveis se a equipe fosse presencial (Fernadez \& Jawadi, 2015). Além disso, as equipes virtuais permitem que as organizações se tornem mais flexíveis e reduzam custos de transação, viagem e transporte, proporcionando significativo aumento da produtividade (Anoye \& Kouame, 2018).

Para conseguir esse salto de produtividade, entretanto, é necessário que os líderes desenvolvam uma plataforma de confiança, harmonia e cooperação incluindo pessoas que, muitas vezes nunca tiveram contato face a face. Um grande desafio para o líder, pois, segundo Fernadez \& Jawadi (2015), para criar esta plataforma são necessários itens como: comunicação abundante, interação pessoal, respeito, honestidade, consenso, reconhecimento de interesse mútuo e coordenação de comportamentos. Ou seja, o líder deve ter competência para gerenciar os relacionamentos, criar um ambiente cooperativo e manter os membros motivados e alinhados com os objetivos da equipe (Anoye \& Kouame, 2018). 


\section{CONSIDERAÇÕES FINAIS}

As equipes virtuais oferecem um modelo para estruturar o trabalho em organizações que desejam alavancar recursos escassos e alcançar as habilidades necessárias, independentemente das limitações geográficas e temporais. Essas equipes são constituídas por indivíduos geograficamente dispersos que usam tecnologias da informação e comunicação (TIC's) para realizar atividades de trabalho. Este artigo procurou identificar os desafios dos líderes e os estilos de liderança mais adequados para manter o bom desempenho de uma equipe virtual.

A partir da análise da literatura identificou-se os principais desafios do líder de equipes virtuais: 1) promover estreita comunicação e cooperação entre membros da equipe; 2) incentivar e reconhecer líderes emergentes; 3 ) estabelecer normas e procedimentos no início da formação e desenvolvimento da equipe; 4) definir limites adequados entre casa e trabalho; 5) Monitorar o progresso da equipe usando as tecnologias; e 6) usar um modelo de controle baseado em objetivos e metas e não por horas.

Quanto ao estilo de liderança, a literatura não aponta um que seja o mais adequado para as equipes virtuais. Entretanto, alguns autores sugerem que os líderes com perfil transformacional podem obter bons resultados nesse tipo de equipe, embora o impacto do líder transformacional sobre a equipe virtual diminua com a dispersão do time (Sedrine, Bouderbala \& Nasraoui, 2020; Eisenberg, Post \& DiTomaso 2019; Mysirlaki \& Paraskeva 2019).

A boa e frequente comunicação e o desenvolvimento de relacionamentos parecem ser mais importantes no contexto das equipes virtuais do que no das equipes tradicionais. De uma certa maneira estas duas características contribuem para criar um "espírito de equipe" no contexto das equipes virtuais cujos membros tendem a se concentrar mais na tarefa e na tecnologia do que nas relações.

Por fim, constatou-se que a maioria dos artigos sobre liderança nas equipes virtuais examina como os líderes usam a tecnologia para interagir virtualmente com os membros da equipe. Existem, entretanto, poucos estudos que tratam da dinâmica da liderança em equipes virtuais. 


\section{REFERÊNCIAS}

Anoye, A., Kouamé, J. (2018). Leadership Challenges in virtual team environment. International Journal of Scientific \& Technology Research.V7, n.7, 2018.

Avolio, B. J., Kahai, S., \& Dodge, G. E. (2001). E-leadership: implications for theory, research, and practice. The Leadership Quarterly, 11(4), 615-668.

Avolio, B. J., Kahai, S., Dumdum, R., \& Sivasubramaniam, N. Virtual teams: implications for e-leadership and team development. In M. London (Ed.), How People Evaluate Others in Organizations (pp. 337-358). Mahwah, NJ: Lawrence Erlbaum, 2001.

Avolio, B., Sosik, J., Kahai, S., \& Baker, B. (2014). E-leadership: Re-examining transformations in leadership source and transmission, The Leadership Quarterly, 25, 105-131, 2014.

Baruch, Y. (2000). Teleworking: benefits and pitfalls as perceived by professionals and managers. New technology, work and employment, 15(1), 34-49.

Botelho, L., Cunha, C., Macedo, M. (2011). O método da revisão integrativa nos estudos organizacionais. Gestão E \& Sociedade, 5(11), 121-136.

Braun, V., Clarke, V. (2006). Using thematic analysis in psychology. Qualitative Research in Psychology. v.3 n.2, p.77-101.

Cascio, W. F. (2000). Managing a virtual workplace. Academy of Management Perspectives, 14(3), 81-90.

Cascio, W., Shurygailo, S. (2003). E-leadership and virtual teams. Organizational Dynamics, vol.31, n.04, pp. 362-376.

Davis, D.; Bryant, J. (2003). Influence at a distance: leadership in global virtual teams. Advances in Global Leadership.

Dulebohn, J., Hoch, J. (2017). Virtual teams in organizations. Human resource management review.

Eisenberg, J., Post, C., \& DiTomaso, N. (2019). Team dispersion and performance: The role of team communication and transformational leadership. Small Group Research, 50(3), 348-380.

Fabbri, S., Octaviano, F., Silva, C., Di Thommazo, A., Hernandes, E., \& Belgamo, A. (2016). Improvements in the Start tool to better support the systematic review process. In Proc. of the 20th International Conference on Evaluation and Assessment in Software Engineering (EASE'16), Limerick, Ireland

Fan, K., Chan, Y., Wang, C., Chen, M. (2014). E-leadership effectiveness in virtual teams: Motivating language perspective. Industrial Management and Data Systems.

Fernandez, D.; Jawadi, N. (2015). Virtual R\&D Project Teams: From E-Leadership To Performance. The Journal of Applied Business Research - Volume 31, N.5.

Forsyth, D. R. Group dynamics. 5. Ed. Wadsworth, Cenage Learnig, 2010. 
Garcia, I. (2020). e-Leadership: A Bibliometric Analysis. International Journal of Advanced Corporate Learning, 13(1), 19-34.

Hambley, L., O'neill, T. Kline, J. (2007). Virtual team leadership: The effects of leadership style and communication medium on team interaction styles and outcomes, Organizational Behavior and Human Decision Processes 103,1-20.

Hickman. A. \& Saad, L. (2020). Reviewing Remote Work in the U.S. Under COVID-19. Gallup. Recuperado a partir de https://news.gallup.com/poll/311375/reviewing-remotework-covid.aspx.

Kratzer, J., Leenders, R. T. A., \& van Engelen, J. M. (2005). Keeping virtual R\&D teams creative. Research-Technology Management, 48(2), 13-16.

Kylefalk, I., \& Hallberg, L. (2020). Multi-Site Leadership: Coordinating and Leading Virtual Teams. Recuperado a partir de https://www.divaportal.org/smash/get/diva2:1448976/FULLTEXT01.pdf

Machado, A. Brandão, C. (2019). Leadership and technology: Concepts and questions Advances in Intelligent Systems and Computing. Volume 931, 2019, Pages 764-773. World Conference on Information Systems and Technologies, WorldCIST 2019; Galicia; Spain; 16 April 2019 through 19 April 2019; Code 224789

Maduka, N.S., Edwards, H., Greenwood, D., Osborne, A., \& Babatunde, S.O. (2018). Analysis of competencies for effective virtual team leadership in building successful organisations. Benchmarking.

Malhotra, A., Majchrzak, A., \& Rosen, B. (2007). Leading virtual teams. Academy of Management perspectives, 21(1), 60-70.

Mcgrath, J. E.; Argote, L. (2001). Group Processes in Organizational Contexts. In: HOGG, M. A.; TINDALE, R. S. Blackwell Handbook of social psychology: Group processes. Blackwell Publishers.

Mysirlaki, S., \& Paraskeva, F. (2019). Virtual team effectiveness: insights from the virtual world teams of massively multiplayer online games. Journal of Leadership Studies, 13(1), 36-55.

Northouse, P. (2004). Leadership: Theory and Practice. 3. Ed. Thousand Oaks: Sage Publications.

O'Mara, M. (2020). Twitter Could End the Office as We Know It. New York equipes. Recuperado a partir de https://www.ny equipes.com/2020/05/19/opinion/twitter-workfrom-home.html

Picu, C. G., \& Dinu, A. (2016). Research on the current telecommuting trends in United States and european union markets. Management and Economics Review, 1(2), 194-202.

Purvanovaa, R., \& Bono, J. Transformational leadership incontext :Face-to-face and virtual teams. The Leadership Quarterly, 20, 343-357, 2009. 
Sedrine, S. B., Bouderbala, A., \& Nasraoui, H. (2020). Leadership style effect on virtual team efficiency: trust, operational cohesion and media richness roles. Journal of Management Development.

Townsend, A. M., DeMarie, S. M., \& Hendrickson, A. R. (1998). Virtual teams: Technology and the workplace of the future. Academy of Management Executive, 12(3), 17-29.

Tranfield, D., Denyer. D., \& Smart. P. (2003) Towards a Methodology for Developing Evidence-Informed Management Knowledge by Means of Systematic Review. British Journal of Management, 14(3):207-222.

Wakefield, R., Leidner, D., \& Garrison, G. (2008). A Model of Conflict, Leadership, and Performance in Virtual Teams. Information Systems Research 19(4):434-455.

Whittemore, R.; Knafl, K. (2005). The integrative review: updated methodology. Journal of advanced nursing, Malden, v. 52, n. 5, p. 546-553.

Yukl, G. Leadership in organizations. 7. Ed. Upper Saddle River: Prentice Hall, 2010.

Zimmermann, P., Wit, A., \& Gill, R. (2008). The relative importance of leadership behaviours in virtual and face-to-face communication settings. Leadership, 4(3), 321-337. 